\title{
Time Course Observation of Outcomes between Minimally Invasive Transforaminal Lumbar Interbody Fusion and Posterior Lumbar Interbody Fusion
}

\author{
Guang-Xun LIN, ${ }^{1}$ Chun-Kun PARK, ${ }^{2}$ Jung-Woo HUR, ${ }^{1}$ and Jin-Sung KIM ${ }^{1}$ \\ ${ }^{1}$ Department of Neurosurgery, Seoul St. Mary's Hospital, The Catholic University of Korea, \\ Seoul, Korea; \\ ${ }^{2}$ Department of Neurosurgery, Good Doctor Teun Teun Hospital, Anyang, Korea
}

\begin{abstract}
The purpose of this study is to compare the long-term patient-outcomes, spinal fusion, and incidence of adjacent segment degeneration (ASD) between minimally invasive transforaminal lumbar interbody fusion (MIS-TLIF) and open posterior lumbar interbody fusion (O-PLIF). We retrospectively reviewed 70 consecutive cases who underwent single-level MIS-TLIF or O-PLIF from March 2010 to July 2013. All the patients achieved a minimum of 5-year follow-up. Data collected for each patient included demographic data, perioperative data, and complications. Clinical outcomes were evaluated with Oswestry disability index and visual analogue scale (VAS). Radiological outcomes included fusion rate and ASD. About 34 patients of MIS-TLIF and 36 patients of O-PLIF were enrolled. Higher Charlson comorbidity index scores were noted in MIS-TLIF than in O-PLIF. Blood loss was significantly lower in MIS-TLIF than O-PLIF. There were significant improvements in clinical and radiological outcomes in both groups. At 6 months, in MIS-TLIF group had significantly lower VAS for back pain and disc height compared with in O-PLIF group. The fusion rate was similar between the two groups at 5-year follow-up. Although the total complication rates were similar between the two groups, both the incidence of ASD was significantly higher in O-PLIF group than MIS-TLIF group $(P=\mathbf{0 . 0 3 2})$. In conclusion, this study indicates that MIS-TLIF is comparable to O-PLIF in terms of fusion rates and clinical outcomes in single-segment degenerative lumbar diseases. In addition, compared with O-PLIF, MIS-TLIF has the advantages of lesser blood loss, faster recovery, and lower incidence of ASD.
\end{abstract}

Key words: minimally invasive transforaminal lumbar interbody fusion, posterior lumbar interbody fusion, adjacent segment degeneration, long-term, degenerative lumbar disease

\section{Introduction}

Spinal fusion surgery is a common technique used to treat degenerative lumbar pathologies including disc herniation with instability, spinal stenosis, and degenerative spondylolisthesis. ${ }^{1-3)}$ Surgery provides sufficient neural decompression and stabilization through interbody fusion for pain relief. Traditionally, posterior lumbar interbody fusion (PLIF) and transforaminal LIF (TLIF) performed through open approach are acceptable and safe options to achieve satisfactory clinical outcomes. ${ }^{4,5)}$ However, it needs extensive paraspinal muscle dissection and retraction. ${ }^{6)}$

Received October 2, 2018; Accepted February 26, 2019

Copyright $@ 2019$ by The Japan Neurosurgical Society This work is licensed under a Creative Commons AttributionNonCommercial-NoDerivatives International License.
The superiority of one procedure over the other still remains controversial according to current literature. Recent development of minimally invasive spine (MIS) surgery for decompression and posterior interbody fusion decreases blood loss and postoperative pain, promotes quick rehabilitation, and leads to shorter hospital stays. ${ }^{7)}$ When indicated, MIS surgery results in adequate bilateral decompression through a unilateral approach. ${ }^{8)}$ However, long-term followup of MIS-TLIF is reported to yield similar results compared with open TLIF/PLIF in terms of fusion rates and complications. ${ }^{9,10)}$

Despite several reports comparing MIS and open procedures, direct comparison of long-term outcomes with MIS-TLIF and open PLIF (O-PLIF) has yet to be reported. The purpose of our study is to compare patient outcomes, spinal fusion, and incidence of complications between MIS-TLIF and O-PLIF for 
treatment of single-level lumbar spine pathology at a minimum of 5-year follow-up.

\section{Materials and Methods}

This retrospective study has been approved by the Institutional Review Board of the authors' affiliated institution. Inclusion criteria were patients with degenerative lumbar disease treated by single-level posterolateral interbody fusion and achieved a minimum of 5-year follow-up. Patients who had trauma, tumor or infection were excluded. At our hospital, patients with degenerative lumbar disease were usually treated by PLIF, and by MIS-TLIF became widely available since 2012. A total of 102 consecutive patients who underwent single-level posterolateral interbody fusion (42 with MIS-TLIF and 60 with O-PLIF) from March 2010 to July 2013. Thirty-two (31.4\%; eight cases in MIS-TLIF and 24 cases in OPLIF) of 102 patients were excluded due to lost follow-up. Finally, 34 patients who underwent single-level MIS-TLIF and 36 with single-level O-PLIF satisfied the aforementioned inclusion criteria.

The surgical procedures are described in previous reports. ${ }^{11,12)}$ Briefly, in both the techniques, decompression of the stenosed segment was followed by interbody fusion with cage. In MIS-TLIF group, under microscopic visualization, a single cage filled with autologous bone graft derived from unilateral total facetectomy and partial laminectomy was inserted. Bilateral decompression through unilateral laminectomy was performed when required. After insertion of single-cage (Crescent, Medtronic Sofamor Danek, Memphis, TN, USA; or Opal, DePuySynthes Spine, Raynham, MA, USA; or Capstone, Medtronic Sofamor Danek), we put percutaneous pedicle screws. In O-PLIF group, the standard fusion procedure was performed after wide decompression of the spinal canal and adjoining foramina, insertion of two cages (Plivios, DePuy-Synthes Spine, or Opal, DePuy-Synthes Spine, or Capstone, Medtronic Sofamor Danek) with autologous bone graft, and insertion of open pedicle screws. There were two spine surgeons who performed O-PLIF procedure whereas the MIS-TLIF was only performed by the senior author.

Patients' demographic data, perioperative data, cage size, Charlson comorbidity index (CCI), ${ }^{13)}$ and complications (perioperative and postoperative) were collected.

Clinical outcomes were evaluated with Oswestry disability index (ODI) and Visual Analogue Scale (VAS) for back and leg pain before surgery and at 6-month, 2- and 5-year follow-up intervals after surgery by an independent research nurse.

Radiographic parameters were measured as follows: pelvic incidence (PI), pelvic tilt (PT), sacral slope (SS), segmental lordotic angle (SLA), lumbar lordotic angle (LLA), disc height (DH), and sagittal vertical axis (SVA) on standing lateral X-ray view. PI was measured as the angle between a line perpendicular to the midpoint of the S1 superior endplate and a line connecting this point to the center of the femoral head axis. PT was measured as the angle between a vertical line from midpoint of the S1 superior endplate and a line connecting this point to the center of the femoral head axis. SS was measured as the angle between a line parallel to the endplate of S1 and a horizontal line. SLA was measured as the angle between the superior endplate of upper level and the inferior endplate of lower level. LLA was the angle between superior endplate of the L1 and superior endplate of sacrum. DH refers to the distance between the inferior and superior endplate of the index level. SVA was defined as the distance between a vertical line from the center of C7 vertebra and a posterior upper corner of S1 vertebra. SVA may be balanced (SVA $<50 \mathrm{~mm}$ ) or imbalanced (SVA $\geq 50$ $\mathrm{mm}$ ) while PI minus lumbar lordosis (PI - LL) was considered appropriate (PI $-\mathrm{LL}<10^{\circ}$ ) or a mismatch (PI - LL $\left.\geq 10^{\circ}\right)^{14,15)}$ All patients underwent repeated $\mathrm{X}$-ray before surgery, immediately after surgery, at 2 and 5 years postoperatively. The cross-sectional area of the spinal canal (CSAC) was calculated in the axial $\mathrm{T}_{2}$-weighted magnetic resonance imaging (MRI) image before surgery. Fusion status was evaluated using the Bridwell criteria ${ }^{16)}$ with computed tomography (CT) at 2 and 5 years, postoperatively. Cage subsidence was deemed to occur if there was evidence of more than $2 \mathrm{~mm}$ migration into the vertebral body on CT.

Adjacent segment degeneration (ASD) was defined on radiographs as the onset of degenerative changes in the previously normal disc spaces adjacent to the fusion segment; along with spondylolisthesis, dynamic instability, or degenerative scoliosis. ${ }^{17)}$

All measurements were done on the picture archives communication system, MaroView 4.5 (Marotech, Seoul, Korea). All the data were evaluated by two senior spine surgeons who were blinded to the situation.

\section{Statistical analysis}

Statistical analysis was performed using the SPSS 23.0 software (IBM, Armonk, NY, USA). Quantitative variables were presented as mean \pm SD (standard deviation) and qualitative variables were expressed in number or percentage. Continuous variables were evaluated by independent sample $t$-test and 
categorical variables evaluated by Chi-squared test or Fisher's exact test. A $P$-value $<0.05$ was considered as statistical significance.

\section{Results}

All the patients consented to participate after more than 5-year follow-up. Table 1 summarizes the demographic characteristics of the patients and intraoperative data. A total of 34 patients $(73.5 \%$ were females) were in MIS-TLIF group and 36 patients (83.3\% were females) in O-PLIF group. The patients' age was lower in O-PLIF group (mean of $59.9 \pm 8.2$ years; range from 45 to 75 years) compared with the MIS-TLIF group (mean of $65.4 \pm 7.6$ years; range from 51 to 81 years $)(P=0.005)$. There was a similar follow-up duration between O-PLIF group (mean of $5.8 \pm 0.9$ years; range from 5 to 8 years) and MIS-TLIF group (mean of $5.4 \pm 0.5$ years; range from 5 to 6 years $)(P=0.057)$. Most of the patients underwent surgery at L4-L5 level in both groups. A few patients manifested degenerative spinal stenosis (two patients accompany by index level instability in MIS-TLIF group and three with instability in O-PLIF group; $P=0.211$ ). The most frequent primary diagnosis was spondylolisthesis $58.8 \%$ in MIS-TLIF and $77.8 \%$ in O-PLIF; $P=0.430$ ) in both groups. There was no significantly different in the degree of spondylolisthesis grade (for Grade II, $14.7 \%$ in MIS-TLIF and $27.8 \%$ in O-PLIF; $P=0.183$ ) between the two groups. Although not reached statistically different, a slightly higher incidence of spondylolisthesis and Grade II spondylolisthesis in O-PLIF group than MIS-TLIF group. For the CCI, a higher score in MIS-TLIF patients compared with O-PLIF patients $(2.6 \pm 1.4$ vs. $1.9 \pm 1.0 ; P=0.012)$ was observed. Gender, body mass index (BMI), BMI $\geq$ $25 \mathrm{~kg} / \mathrm{m}^{2}$, bone mineral density (BMD), $\mathrm{BMD} \leq-2.5$, and CSAC were not significantly different between the groups $(P>0.05)$. Additionally, 14 out of 34 patients $(41.2 \%)$ in MIS-TLIF group underwent bilateral decompression through unilateral approach.

Estimated blood loss was significantly lower in the MIS-TLIF group (mean of $225.2 \pm 94.1 \mathrm{~mL}$ ) compared with O-PLIF (mean of $509.1 \pm 138.6 \mathrm{~mL}$ ) $(P<0.001)$. No significant difference in operative times and postoperative hospitalization between the two groups was noted $(P>0.05)$.

Seven out of 34 patients in MIS-TLIF group manifested eight complications. One patient who had two complications (dural tear and pulmonary thromboembolism) was referred to and treated by the Department of Cardiology. One patient had symptomatic screw malposition, which required revision surgery. One patient had bone graft dislodgement,
Table 1 Comparison of demographic, intraoperative data, and complications between the two groups

\begin{tabular}{|c|c|c|c|}
\hline & $\begin{array}{l}\text { MIS-TLIF } \\
(n=34)\end{array}$ & $\begin{array}{l}\text { O-PLIF } \\
(n=36)\end{array}$ & $P$ \\
\hline $\begin{array}{l}\text { Gender ratio } \\
\text { (Male/Female) }\end{array}$ & $9: 25$ & $6: 30$ & 0.318 \\
\hline Diagnosis & & & 0.088 \\
\hline $\begin{array}{l}\text { Spondylolisthesis } \\
\text { (Grade\# I vs. II) }\end{array}$ & $20(15: 5)$ & $28(18: 10)$ & \\
\hline $\begin{array}{l}\text { Spinal Stenosis } \\
\text { (with vs. without } \\
\text { instability) }\end{array}$ & $14(2: 12)$ & $8(3: 5)$ & \\
\hline $\begin{array}{l}\text { Age (years); } \\
\text { mean } \pm \text { SD }\end{array}$ & $65.4 \pm 7.6$ & $59.9 \pm 8.2$ & $0.005^{*}$ \\
\hline $\begin{array}{l}\mathrm{BMI}\left(\mathrm{kg} / \mathrm{m}^{2}\right) \\
\text { mean } \pm \mathrm{SD}\end{array}$ & $24.7 \pm 2.5$ & $24.2 \pm 3.1$ & 0.478 \\
\hline $\begin{array}{l}\mathrm{BMI} \geq 25 \mathrm{~kg} / \mathrm{m}^{2} ; \\
n(\%)\end{array}$ & $15(44.1 \%)$ & $13(36.1 \%)$ & 0.494 \\
\hline $\mathrm{BMD} ;$ mean $\pm \mathrm{SD}$ & $-1.8 \pm 1.2$ & $-2.2 \pm 1.1$ & 0.169 \\
\hline $\mathrm{BMD} \leq-2.5 ; n(\%)$ & $11(32.4 \%)$ & $15(41.7 \%)$ & 0.420 \\
\hline $\begin{array}{l}\text { CCI (points); } \\
\text { mean } \pm \text { SD }\end{array}$ & $2.6 \pm 1.4$ & $1.9 \pm 1.0$ & $0.012^{*}$ \\
\hline Operative level & & & 0.582 \\
\hline L3-L4 & 1 & 2 & \\
\hline L4-L5 & 22 & 26 & \\
\hline L5-S1 & 11 & 8 & \\
\hline $\begin{array}{l}\mathrm{CSAC}\left(\mathrm{mm}^{2}\right) \\
\text { mean } \pm \mathrm{SD}\end{array}$ & $113.8 \pm 72.3$ & $116.1 \pm 86.6$ & 0.904 \\
\hline $\begin{array}{l}\text { Duration of } \\
\text { follow-up (years); } \\
\text { mean } \pm \text { SD }\end{array}$ & $5.4 \pm 0.5$ & $5.8 \pm 0.9$ & 0.057 \\
\hline $\begin{array}{l}\text { Operative times } \\
\text { (min); mean } \pm \text { SD }\end{array}$ & $167.1 \pm 39.1$ & $155.9 \pm 25.7$ & 0.207 \\
\hline $\begin{array}{l}\text { Estimated blood } \\
\text { loss (mL); } \\
\text { mean } \pm \text { SD }\end{array}$ & $225.2 \pm 94.1$ & $509.1 \pm 138.6$ & $<0.001^{*}$ \\
\hline $\begin{array}{l}\text { Postoperative } \\
\text { hospitalization } \\
\text { (days); mean } \pm \text { SD }\end{array}$ & $7.8 \pm 2.3$ & $8.4 \pm 5.3$ & 0.592 \\
\hline $\begin{array}{l}\text { Adjacent segment } \\
\text { degeneration; } n(\%)\end{array}$ & $4(11.8 \%)$ & $12(33.3 \%)$ & $0.032^{*}$ \\
\hline $\begin{array}{l}\text { Total complication } \\
\text { rates; \% }(n)\end{array}$ & $23.5 \%(8 / 34)$ & $38.9 \%(14 / 36)$ & 0.167 \\
\hline
\end{tabular}

${ }^{*} P<0.05$. "The grade of spondylolisthesis is assessed by using Meyerding classification. BMD: bone mineral density, BMI: body mass index, CCI: charlson comorbidity index, CSAC: cross-sectional area of the spinal canal, MIS-TLIF: minimally invasive transforaminal lumbar interbody fusion, O-PLIF: open posterior lumbar interbody fusion.

with no neurological sequelae. There were four cases of ASD, none of which required surgery. Fourteen out of 36 patients in O-PLIF group had 14 complications. One patient developed epidural 
hematoma, which required emergency surgical evacuation. One asymptomatic patient with osteolysis was managed by observation alone. There were 12 patients observed ASD, four of whom required surgery to address the clinical symptoms caused by ASD. One patient underwent O-PLIF revision surgery, two patients underwent MIS-TLIF revision surgery (Fig. 1), and one underwent minimally invasive discectomy by Disc-FX. ${ }^{18)}$ Although the total complication rates were a slightly lower in MIS-TLIF group compared with O-PLIF group (23.5\% vs. $38.9 \%$ ), there was no statistically significant difference between them $(P=0.167)$. The incidence of ASD was significantly higher in O-PLIF group than in MIS-TLIF $(P=0.032)$ (Table 1).

In O-PLIF group, the heights of the used cage were $10 \mathrm{~mm}$ in three levels, $11 \mathrm{~mm}$ in 10 levels, $12 \mathrm{~mm}$ in 20 levels, and $14 \mathrm{~mm}$ in three levels; cage lordotic angle was $4^{\circ}$ in five levels, $6^{\circ}$ in one level, and $8^{\circ}$ in one level. In MIS-TLIF group, the heights of the used cage were $8 \mathrm{~mm}$ in three levels, $10 \mathrm{~mm}$ in 23 levels, $12 \mathrm{~mm}$ in four levels, and $14 \mathrm{~mm}$ in four levels; cage lordotic angle was $6^{\circ}$ in seven levels. The mean cage height was significantly higher in MIS-TLIF group $(11.7 \pm 0.9 \mathrm{~mm})$ compared with O-PLIF (10.5 \pm $1.6 \mathrm{~mm})(P<0.001)$.

The mean ODI scores showed significant improvement, from preoperative $48.6 \pm 9.4$ to $25.7 \pm 8.2$, 21.1 \pm 9.0 , and $19.9 \pm 10.9$ at postoperative 6 months, 2 and 5 years in O-PLIF group, respectively; and from $50.8 \pm 9.7$ to $28.9 \pm 6.5,19.9 \pm 7.3$, and $17.1 \pm 8.1$ in MIS-TLIF group, respectively. VAS for back pain was, significantly lower in MIS-TLIF than in O-PLIF patients at 6 months postoperatively, with a mean score of $2.8 \pm 0.9$ vs. $3.5 \pm 1.2(P=0.014)$, respectively (Fig. 2). In terms of clinical outcomes before and after surgery, the ODI and VAS for back and VAS for leg demonstrated a significant improvement in both groups $(P<0.05)$, with $66.3 \%, 70.5 \%$, and $72.5 \%$ improvement in MIS-TLIF group compared with $58.8 \%, 66.7 \%$, and $67.1 \%$ improvement in O-PLIF group, respectively, with no statistically significant differences between the two groups $(P>0.05)$.

Pelvic incidence, PT, SS, SVA, and PI - LL were similar between the two groups, without any statistically significant difference from baseline
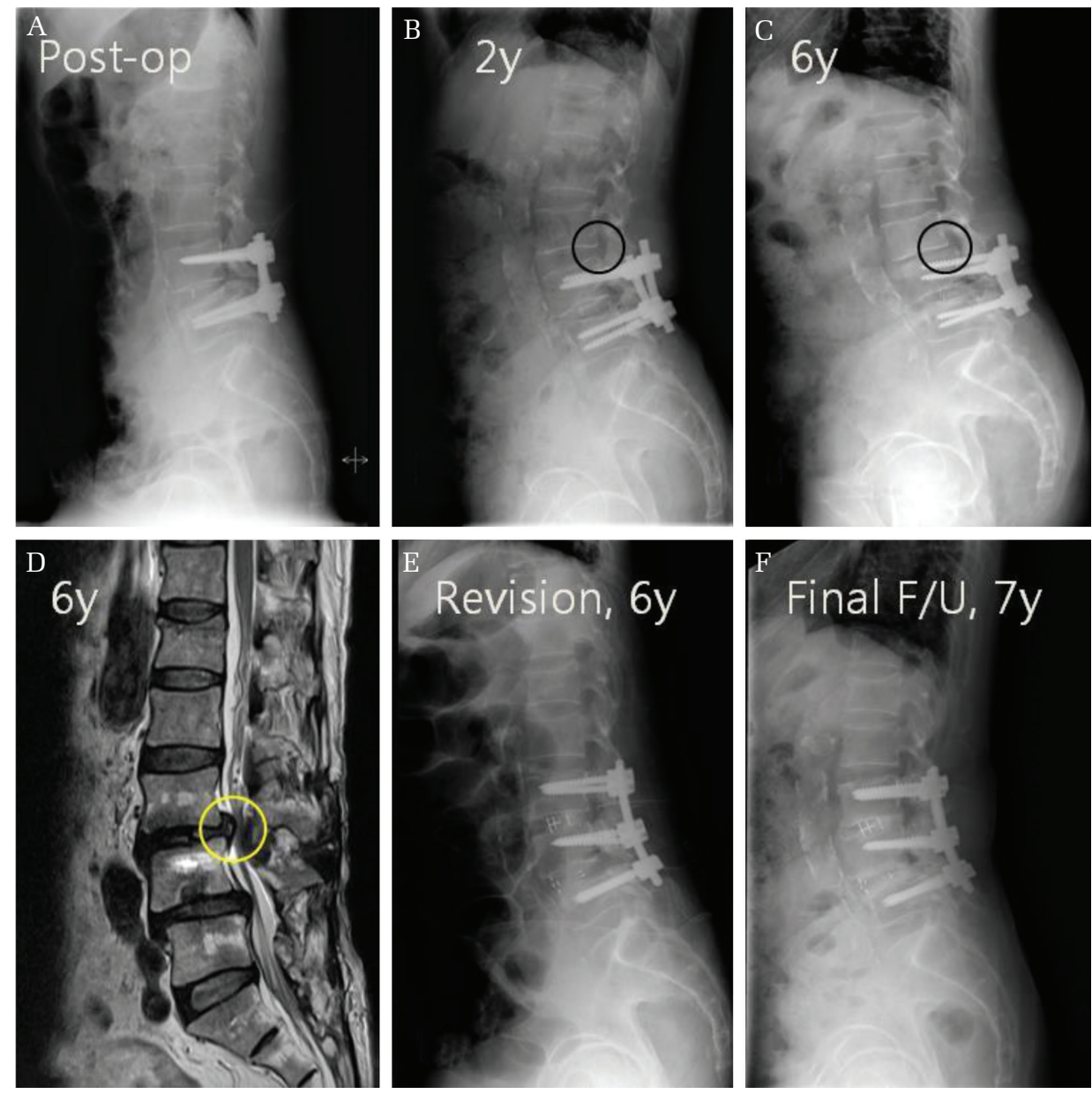

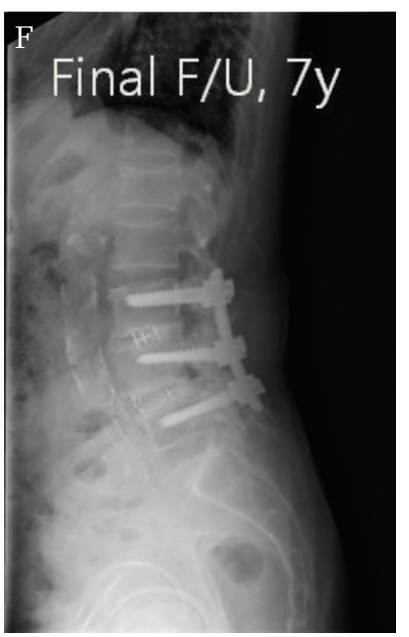

Fig. 1 A 66-year-old female. Postoperative (A), and progressive changes 2 years $(B)$ and 6 years (C and D) after posterior lumbar interbody fusion (PLIF). Revised minimally invasive transforaminal interbody fusion at L3-4 and minimally-access PLIF at L4-L5. After revision (E) and final follow-up (F). 

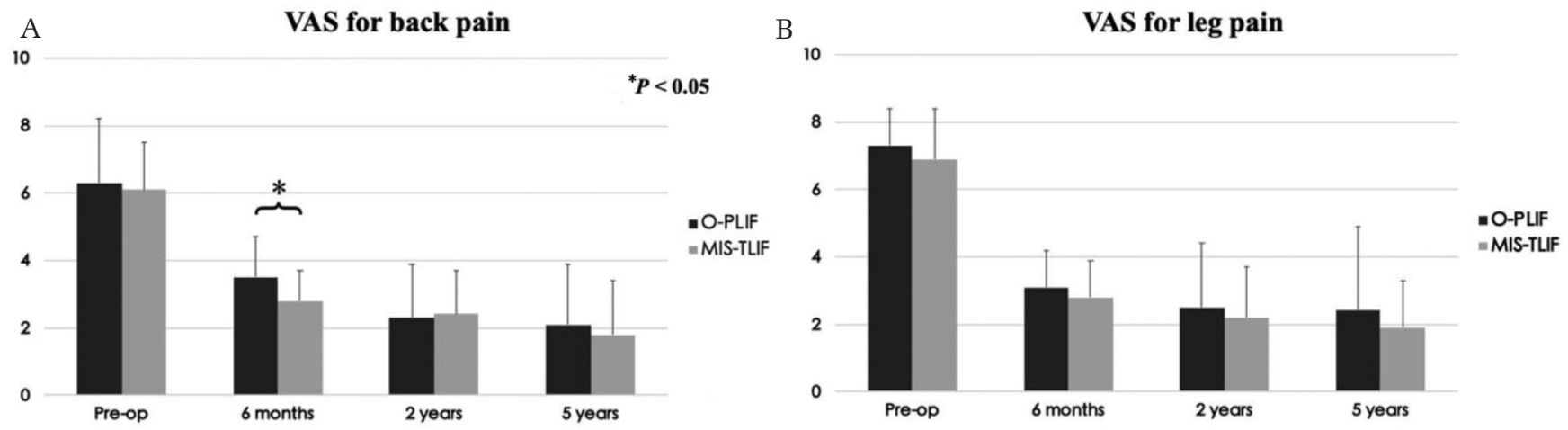

Fig. 2 Visual analogue scale (VAS) scores for leg pain (A) and back pain (B) with time. O-PLIF, open posterior lumbar interbody fusion; MIS-TLIF, minimally invasive spine transforaminal lumbar interbody fusion.

to postoperative values. In both groups, the rate of SVA imbalance and PI - LL mismatch were slightly lower postoperatively than preoperatively, without any statistically significant difference between the two groups $(P>0.05$; Table 2$)$.

Segmental lordotic angle and lumbar lordotic angle were within the normal range preoperatively and remained essentially similar postoperatively. There was no statistical difference between the two groups at long-term follow-up (Fig. 3A). DH was significantly increased postoperatively in both groups, and persisted at long-term follow-up with no statistically significant difference (Fig. 3B). At 6 months, there was a significantly greater $\mathrm{DH}$ in O-PLIF $(12.6 \pm 1.9 \mathrm{~mm})$ compared with MIS-TLIF (11.4 $\pm 2.4 \mathrm{~mm} ; P=0.017)$.

Fusion was achieved in $91.2 \%(31 / 34)$ and $94.1 \%$ (32/34) of the patients in MIS-TLIF group at 2- and 5 -year follow-up, respectively. The fusion rate was $86.1 \%(31 / 36)$ and $91.7 \%(33 / 36)$ in O-PLIF group during the same follow-up time, respectively. There was a slightly higher fusion rate in MIS-TLIF compared with O-PLIF, without any statistical difference between the two groups (2-year follow-up, $P=0.506$; 5-year follow-up, $P=0.691$ ). Overall, five cases of pseudoarthrosis (two cases in MIS-TLIF and three cases in O-PLIF) were observed. Four of these five patients underwent revision surgery due to clinical symptoms, and another patient continued to be observed at the outpatient visit. In addition, 10 cases of cage subsidence were seen in the MIS-TLIF group (29.4\%) 5 years after surgery and nine cases of cage subsidence were seen in the O-PLIF group (25.0\%). There was no statistical difference between the two groups $(P=0.678)$.

\section{Discussion}

Degenerative lumbar diseases causing spinal stenosis and degenerative spondylolisthesis are frequently
Table 2 Comparison of pre- and postoperative radiographic parameters between the two groups

\begin{tabular}{llccc}
\hline & & MIS-TLIF & O-PLIF & $P$ \\
\hline PI ( $\left.{ }^{\circ}\right)$ & Pre-op & $53.2 \pm 8.0$ & $52.7 \pm 9.2$ & 0.795 \\
& Post-op & $53.4 \pm 7.8$ & $52.8 \pm 9.1$ & 0.786 \\
PT ( $\left.{ }^{\circ}\right)$ & Pre-op & $23.5 \pm 8.8$ & $22.9 \pm 5.8$ & 0.719 \\
& Post-op & $24.4 \pm 9.4$ & $23.0 \pm 5.7$ & 0.419 \\
SS ( $\left.{ }^{\circ}\right)$ & Pre-op & $29.2 \pm 6.3$ & $29.0 \pm 8.0$ & 0.919 \\
& Post-op & $28.9 \pm 6.4$ & $28.7 \pm 8.0$ & 0.914 \\
SVA (mm) & Pre-op & $41.9 \pm 26.8$ & $37.3 \pm 33.2$ & 0.502 \\
& Post-op & $31.4 \pm 18.9$ & $30.6 \pm 22.1$ & 0.855 \\
SVA $\geq$ & Pre-op & $29.4 \%(10 / 34)$ & $33.3 \%(12 / 36)$ & 0.724 \\
50 mm (\%) & Post-op & $20.6 \%(7 / 34)$ & $25.0 \%(9 / 36)$ & 0.660 \\
PI - LL $\geq$ & Pre-op & $44.1 \%(15 / 34)$ & $52.8 \%(19 / 36)$ & 0.469 \\
$10^{\circ}(\%)$ & Post-op & $38.2 \%(13 / 34)$ & $44.4 \%(16 / 36)$ & 0.598 \\
\hline
\end{tabular}

MIS-TLIF: minimally invasive transforaminal lumbar interbody fusion, O-PLIF: open posterior lumbar interbody fusion, PI: pelvic incidence, PI - LL: pelvic incidence minus lumbar lordosis, Post-op: postoperative, Pre-op: preoperative, PT: pelvic tilt, SS: sacral slope, SVA: sagittal vertical axis.

associated with chronic low back pain, radicular symptoms, and spinal instability. ${ }^{19)}$ Numerous options for fusion are available to treat lumbar spinal stenosis and spondylolisthesis, to improve pain and disability. The popularity of O-PLIF over the past decades has been attributed to improved clinical satisfaction and corrective radiographic outcomes after surgery. ${ }^{20)}$ However, retraction of dural and nerve roots in O-PLIF during discectomy and cage insertion is a drawback. This manipulation increases the risk of related complications. MIS-TLIF technique is an alternative and modified version of O-PLIF, which facilitates direct nerve root decompression in addition to interbody fusion. MIS-TLIF leads to satisfactory 

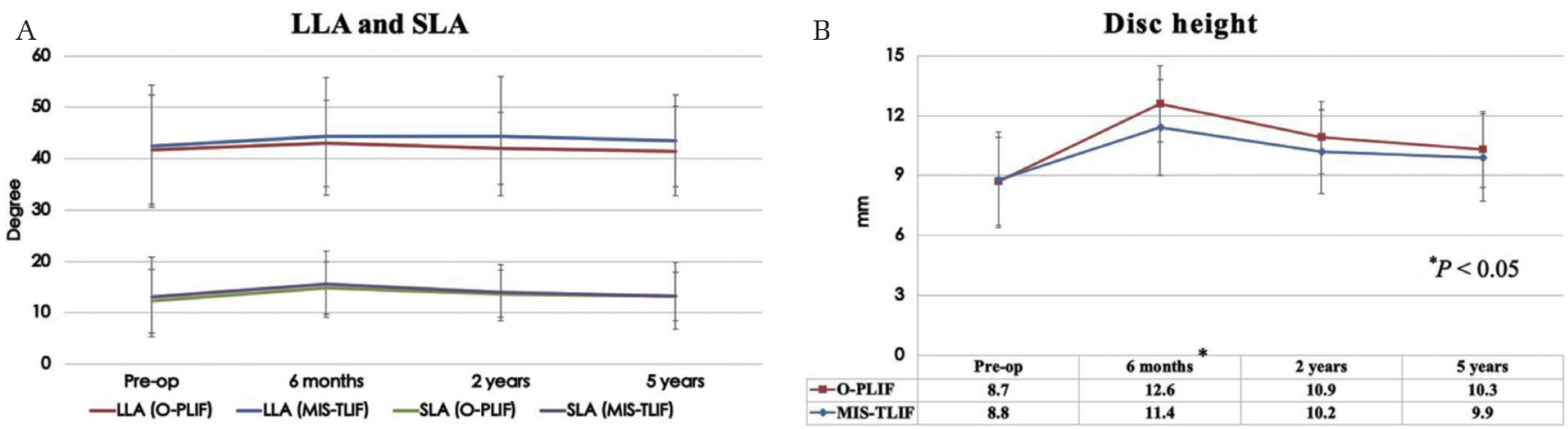

Fig. 3 (A) Segmental lordotic angle (SLA) and lumbar lordotic angle (LLA) with time. (B) Disc height (DH) with time. O-PLIF, open posterior lumbar interbody fusion; MIS-TLIF, minimally invasive spine transforaminal lumbar interbody fusion.

clinical and radiological outcomes, which are well documented. ${ }^{21,22)}$

To the best of our knowledge, our report represents the first long-term follow-up study directly comparing patient outcomes, spinal fusion, and incidence of complications associated with MIS-TLIF and O-PLIF procedures for treatment of single-level degenerative pathology.

In our study, most of the patients were diagnosed with degenerative spondylolisthesis, and a few patients manifested degenerative spinal stenosis, which required fusion surgery. There was none of patients with degenerative lumbar scoliosis. Benefits of MIS-TLIF include smaller incision, minimal paravertebral muscle damage, and less destruction of bone structures. These factors may result in the reduced estimated blood loss that corresponds with our result. A similar study analyzed the differences between MIS-TLIF and open PLIF in patients with single-level spondylolisthesis, using bilateral tubular retractors with two cages in all the MIS-TLIF cases. ${ }^{23)}$ They also reported similar fusion rates, shorter hospital stays and operative times, and minimal blood loss in MIS-TLIF group compared with the open PLIF group. However, all of the MIS-TLIF cases in our study were implanted with a single cage. Additionally, $41.2 \%$ of MIS-TLIF patients underwent bilateral decompression through unilateral approach leading to an increase in the mean operative times. This may explain that MIS-TLIF (167.1 $\pm 39.1 \mathrm{~min})$ had a slightly longer operative times than O-PLIF (155.9 $\pm 25.7 \mathrm{~min})$, although statistical differences were not achieved.

The use of one or two cages has no impact on fusion rate or clinical results, although two cages prevent the loss of $\mathrm{DH}$ and correction of lumbar lordosis. ${ }^{24)}$ In this study, MIS-TLIF with a single cage resulted in superior DH restoration postoperatively compared with O-PLIF with two cages involving earlier follow-up. The O-PLIF procedure usually requires retraction of the disc space for insertion of the cage, whereas cage insertion in MIS-TLIF can be achieved through the available disc space height. Although preoperative $\mathrm{DH}$ was similar between the two groups, the differences in methodology may have resulted in inconsistent disc height at earlier postoperative follow-ups. In addition, the mean height of the cage used in O-PLIF group was significantly higher than MIS-TLIF group (11.7 $\pm 0.9 \mathrm{~mm}$ vs. 10.5 $\pm 1.6 \mathrm{~mm} ; P<0.001)$. The contribution of the height of the inserted cage for restoration of the postoperative disc height cannot be ignored. However, there was more cage subsidence in the O-PLIF group, which ultimately led to similar $\mathrm{DH}$ between the two groups at long-term follow-ups. Therefore, surgical techniques are superior to the number of cages used. Segmental and whole lumbar lordosis, which was within the normal range before surgery, remained mostly unchanged after surgery, with no statistically significant difference postoperatively in both groups compared with preoperative values.

The precise pathophysiology of ASD remains unclear despite several studies investigating the mechanisms of pathogenesis. Theoretically, spinal fusion leads to ASD due to biomechanical changes including increased intradiscal pressure, stress at adjacent segmental levels, and increased adjacent segmental motion, which exacerbate degenerative changes. ${ }^{25)}$ These changes are triggered by demographic factors, spinopelvic sagittal balance parameters, or other causes. ${ }^{26,27)}$ The incidence of ASD depends on a variety of factors. Previous literature has reported that the risk factors of ASD include age, gender, BMI ( $\left.\geq 25 \mathrm{~kg} / \mathrm{m}^{2}\right)$, osteoporosis, preoperative PT $\left(>20^{\circ}\right)$, preoperative SS $\left(<40^{\circ}\right)$, preoperative and postoperative lower LL, SVA imbalance, and PI - LL mismatch, etc. ${ }^{28-31)}$ 
In this study, there was no significant differences in terms of gender, BMI, BMD, operated level, and the pre and postoperative spinopelvic sagittal balance parameters. However, a significantly lower incidence of ASD was observed in MIS-TLIF patients compared with O-PLIF patients. A possible explanation is that the MIS-TLIF procedure, compared with O-PLIF, may cause lesser disruption of adjacent segment soft tissue, as well as less destruction of the facet joint. Partial decompressive laminectomy was performed in MIS-TLIF procedure preserves the posterior ligamentous complex to maintain spinal stability. ${ }^{22}$ Furthermore, the factors that most influence these differences depend on the use of percutaneous screws which has a great influence on adjacent facet joint violations and muscle damage that affect the occurrence of ASD (MIS-TLIF with percutaneous screws and O-PLIF with open screws). ${ }^{33)}$ Besides, MIS-TLIF has advantages of reduced multifidus atrophy which is an important reason for the lower frequency of ASD. ${ }^{34)}$ In addition, although not reached statistically different, there was a slightly higher incidence in females $(83.3 \%)$, spondylolisthesis $(77.8 \% ; 27.8 \%$ are Grade II), BMD $\leq-2.5(41.7 \%)$, pre $(33.3 \%)$ and postoperative $(25.0 \%) \mathrm{SVA} \geq 50 \mathrm{~mm}$, and pre $(52.8 \%)$ and postoperative $(44.4 \%)$ PI - LL $\geq 10^{\circ}$ in O-PLIF group compared with MIS-TLIF group which was presenting as female $(73.5 \%)$, spondylolisthesis (58.8\%; $14.7 \%$ are Grade II), BMD $\leq-2.5$ (32.4\%), pre $(29.4 \%)$ and postoperative $(20.6 \%)$ SVA $\geq$ $50 \mathrm{~mm}$, and pre $(44.1 \%)$ and postoperative $(38.2 \%)$ PI - LL $\geq 10^{\circ}$.

There is still controversy about whether there is an association between age and ASD. In theory, the older spine is less flexible and difficult to adapt to the biomechanical changes after fusion, in stark contrast to the young spine. Some studies have indicated that the increase in age was proportional to the incidence of ASD. ${ }^{35,36)}$ However, some studies have reported no association between age and ASD. ${ }^{37{ }^{38)}}$ In this study, although the mean age and CCI scores of MIS-TLIF patients were higher than O-PLIF patients, the incidence of ASD in MIS-TLIF was significantly lower than that of O-PLIF. Hence, we speculate that age is not associated with the onset of ASD. Meanwhile, comorbidities may not be a risk factor for ASD. Perhaps compared with those patient-specific factors, direct destructive damage from surgery may result in a greater likelihood of ASD.

Although higher incidence of ASD in O-PLIF group compared with MIS-TLIF group, clinical outcomes significantly improved in both groups at long-term follow-up with no significant differences between the two groups. However, VAS for back pain was significantly lower in MIS-TLIF patients compared with O-PLIF patients at early time follow-up (only at 6 months postoperatively). This demonstrates that MIS-TLIF results in faster improvement of back pain due to less trauma to soft tissues and preservation of the midline musculo-ligamentous complex during the surgery. In addition, with the exception of one case, all cases of ASD occurred within 4 years after surgery and all reoperation for symptomatic ASD were conducted more than 5 years after the first surgery. These findings displayed that degeneration is a relatively protracted process, and the impact on clinical outcomes during this process is not significant. Therefore, we speculated that the impact of ASD on clinical outcome after fusion surgery does not show a significant correlation, which was consistent with the previous studies. ${ }^{39,40)}$ However, some reports found that it did not necessarily correlate with clinical symptoms. ${ }^{34,41)}$

There are some limitations in this study. First, it is a retrospective nature study with a small sample size. Second, there may be a selection bias because the selection was based only on the date. At last, it may influence the whole results with loss follow-up of the patients from the original surgery populations, which was more than $30 \%$. In future, a multicenter prospective randomized study with a higher number of patients and longer follow-up duration is needed to corroborate the study findings.

\section{Conclusion}

This study demonstrates that MIS-TLIF is comparable to O-PLIF in terms of fusion rate and clinical outcomes for single-segment degenerative lumbar diseases. Moreover, compared with O-PLIF, MIS-TLIF has the merits of less estimated blood loss, quicker improvement of back pain, and lower incidence of ASD. In addition, we speculate that age and comorbidities may not be risk factors for ASD. Concurrently, ASD is not significantly correlated with clinical outcomes.

\section{Acknowledgments}

The authors wish to acknowledge the financial support of the Catholic Medical Center Research Foundation made in the program year of 2016; China Scholarship Council (CSC, No. [2016]3100).

\section{Conflicts of Interest Disclosure}

None of the authors have any conflicts of interest to declare. 


\section{References}

1) Madan S, Boeree NR: Outcome of posterior lumbar interbody fusion versus posterolateral fusion for spondylolytic spondylolisthesis. Spine (Phila Pa 1976) 27: 1536-1542, 2002

2) Schwender JD, Holly LT, Rouben DP, Foley KT: Minimally invasive transforaminal lumbar interbody fusion (TLIF): technical feasibility and initial results. J Spinal Disord Tech 18 Suppl: S1-S6, 2005

3) Wang J, Zhou Y, Zhang ZF, Li CQ, Zheng WJ, Liu J: Comparison of one-level minimally invasive and open transforaminal lumbar interbody fusion in degenerative and isthmic spondylolisthesis grades 1 and 2. Eur Spine J 19: 1780-1784, 2010

4) de Kunder SL, van Kuijk SMJ, Rijkers K, et al.: Transforaminal lumbar interbody fusion (TLIF) versus posterior lumbar interbody fusion (PLIF) in lumbar spondylolisthesis: a systematic review and meta-analysis. Spine J 17: 1712-1721, 2017

5) Yan DL, Pei FX, Li J, Soo CL: Comparative study of PILF and TLIF treatment in adult degenerative spondylolisthesis. Eur Spine J 17: 1311-1316, 2008

6) Humphreys SC, Hodges SD, Patwardhan AG, Eck JC, Murphy RB, Covington LA: Comparison of posterior and transforaminal approaches to lumbar interbody fusion. Spine (Phila Pa 1976) 26: 567-571, 2001

7) Lee KH, Yue WM, Yeo W, Soeharno H, Tan SB: Clinical and radiological outcomes of open versus minimally invasive transforaminal lumbar interbody fusion. Eur Spine J 21: 2265-2270, 2012

8) Than KD, Mummaneni PV: Unilateral approach for bilateral decompression with MIS TLIF. World Neurosurg 82: 646-647, 2014

9) Jin-Tao Q, Yu T, Mei W, et al.: Comparison of MIS vs. open PLIF/TLIF with regard to clinical improvement, fusion rate, and incidence of major complication: a meta-analysis. Eur Spine J 24: 1058-1065, 2015

10) Seng C, Siddiqui MA, Wong KP, et al.: Five-year outcomes of minimally invasive versus open transforaminal lumbar interbody fusion: a matchedpair comparison study. Spine (Phila Pa 1976) 38: 2049-2055, 2013

11) Lin GX, Quillo-Olvera J, Jo HJ, et al.: Minimally invasive transforaminal lumbar interbody fusion: a comparison study based on end plate subsidence and cystic change in individuals older and younger than 65 years. World Neurosurg 106: 174-184, 2017

12) Sivaraman A, Altaf F, Jalgaonkar A, et al.: Prospective study of posterior lumbar interbody fusion with either interbody graft or interbody cage in the treatment of degenerative spondylolisthesis. J Spinal Disord Tech 28: E467-E471, 2015

13) Charlson M, Szatrowski TP, Peterson J, Gold J: Validation of a combined comorbidity index. J Clin Epidemiol 47: 1245-1251, 1994
14) Schwab FJ, Blondel B, Bess S, et al.: Radiographical spinopelvic parameters and disability in the setting of adult spinal deformity: a prospective multicenter analysis. Spine (Phila Pa 1976) 38: E803-E812, 2013

15) Inami $S$, Moridaira $H$, Takeuchi D, Shiba $Y$, Nohara Y, Taneichi H: Optimum pelvic incidence minus lumbar lordosis value can be determined by individual pelvic incidence. Eur Spine J 25: 3638-3643, 2016

16) Bridwell KH, O’Brien MF, Lenke LG, Baldus C, Blanke K: Posterior spinal fusion supplemented with only allograft bone in paralytic scoliosis. Does it work? Spine (Phila Pa 1976) 19: 2658-2666, 1994

17) Hilibrand AS, Robbins M: Adjacent segment degeneration and adjacent segment disease: the consequences of spinal fusion? Spine J 4: 190S-194S, 2004

18) Kumar N, Kumar A, Siddharth MS, Sambhav PS, Tan J: Annulo-nucleoplasty using Disc-FX in the management of lumbar disc pathology: early results. Int J Spine Surg 8: 18, 2014

19) Taher F, Essig D, Lebl DR, et al.: Lumbar degenerative disc disease: current and future concepts of diagnosis and management. Adv Orthop 2012: 970752, 2012

20) Cheung NK, Ferch RD, Ghahreman A, Bogduk N: Long-term follow-up of minimal-access and open posterior lumbar interbody fusion for spondylolisthesis. Neurosurgery 72: 443-450; discussion 450-451, 2013

21) Rouben D, Casnellie M, Ferguson M: Long-term durability of minimal invasive posterior transforaminal lumbar interbody fusion: a clinical and radiographic follow-up. J Spinal Disord Tech 24: 288-296, 2011

22) Peng CW, Yue WM, Poh SY, Yeo W, Tan SB: Clinical and radiological outcomes of minimally invasive versus open transforaminal lumbar interbody fusion. Spine (Phila Pa 1976) 34: 1385-1389, 2009

23) Zhang D, Mao K, Qiang X: Comparing minimally invasive transforaminal lumbar interbody fusion and posterior lumbar interbody fusion for spondylolisthesis: a STROBE-compliant observational study. Medicine (Baltimore) 96: e8011, 2017

24) Aoki Y, Yamagata M, Ikeda Y, et al.: A prospective randomized controlled study comparing transforaminal lumbar interbody fusion techniques for degenerative spondylolisthesis: unilateral pedicle screw and 1 cage versus bilateral pedicle screws and 2 cages. J Neurosurg Spine 17: 153-159, 2012

25) Zhang C, Berven SH, Fortin M, Weber MH: Adjacent segment degeneration versus disease after lumbar spine fusion for degenerative pathology: a systematic review with meta-analysis of the literature. Clin Spine Surg 29: 21-29, 2016

26) Saavedra-Pozo FM, Deusdara RA, Benzel EC: Adjacent segment disease perspective and review of the literature. Ochsner J 14: 78-83, 2014 
27) Park P, Garton HJ, Gala VC, Hoff JT, McGillicuddy JE: Adjacent segment disease after lumbar or lumbosacral fusion: review of the literature. Spine (Phila Pa 1976) 29: 1938-1944, 2004

28) Ould-Slimane M, Lenoir T, Dauzac C, et al.: Influence of transforaminal lumbar interbody fusion procedures on spinal and pelvic parameters of sagittal balance. Eur Spine J 21: 1200-1206, 2012

29) Liang J, Dong Y, Zhao H: Risk factors for predicting symptomatic adjacent segment degeneration requiring surgery in patients after posterior lumbar fusion. J Orthop Surg Res 9: 97, 2014

30) Matsumoto T, Okuda S, Maeno T, et al.: Spinopelvic sagittal imbalance as a risk factor for adjacentsegment disease after single-segment posterior lumbar interbody fusion. J Neurosurg Spine 26: 435-440, 2017

31) Ou CY, Lee TC, Lee TH, Huang YH: Impact of body mass index on adjacent segment disease after lumbar fusion for degenerative spine disease. Neurosurgery 76: 396-401; discussion 401-402; quiz 402, 2015

32) Vazan M, Gempt J, Meyer B, Buchmann N, Ryang YM: Minimally invasive transforaminal lumbar interbody fusion versus open transforaminal lumbar interbody fusion: a technical description and review of the literature. Acta Neurochir (Wien) 159: 1137-1146, 2017

33) Zeng ZL, Jia L, Xu W, et al.: Analysis of risk factors for adjacent superior vertebral pedicle-induced facet joint violation during the minimally invasive surgery transforaminal lumbar interbody fusion: a retrospective study. Eur J Med Res 20: 80, 2015

34) Zhu HF, Wang GL, Zhou ZJ, Fan SW: Prospective study of long-term effect between multifidus muscle bundle and conventional open approach in one-level posterior lumbar interbody fusion. Orthop Surg 10: 296-305, 2018
35) Min JH, Jang JS, Jung BJ, et al.: The clinical characteristics and risk factors for the adjacent segment degeneration in instrumented lumbar fusion. J Spinal Disord Tech 21: 305-309, 2008

36) Cho KS, Kang SG, Yoo DS, Huh PW, Kim DS, Lee SB: Risk factors and surgical treatment for symptomatic adjacent segment degeneration after lumbar spine fusion. J Korean Neurosurg Soc 46: 425-430, 2009

37) Kim KH, Lee SH, Shim CS, et al.: Adjacent segment disease after interbody fusion and pedicle screw fixations for isolated L4-L5 spondylolisthesis: a minimum five-year follow-up. Spine (Phila Pa 1976) 35: 625-634, 2010

38) Zhong ZM, Deviren V, Tay B, Burch S, Berven SH: Adjacent segment disease after instrumented fusion for adult lumbar spondylolisthesis: incidence and risk factors. Clin Neurol Neurosurg 156: 29-34, 2017

39) Yang JY, Lee JK, Song HS: The impact of adjacent segment degeneration on the clinical outcome after lumbar spinal fusion. Spine (Phila $\mathrm{Pa}$ 1976) 33: 503-507, 2008

40) Chen BL, Wei FX, Ueyama K, et al.: Adjacent segment degeneration after single-segment PLIF: the risk factor for degeneration and its impact on clinical outcomes. Eur Spine J 20: 1946-1950, 2011

41) Okuda S, Oda T, Miyauchi A, et al.: Lamina horizontalization and facet tropism as the risk factors for adjacent segment degeneration after PLIF. Spine (Phila Pa 1976) 33: 2754-2758, 2008

Address reprint requests to: Jin-Sung Kim $\mathrm{MD}, \mathrm{PhD}$, Department of Neurosurgery, Seoul St. Mary's Hospital, The Catholic University of Korea, 222 Banpo-daero Seocho-gu, Seoul 06591, South Korea e-mail: mdlukekim@gmail.com 